\title{
EFFECT OF REPEATED HEAT PRESSING AND THERMOCYCLING ON MICRO SHEAR BOND STRENGTH OF POLYETHERETHERKE- TONE (PEEK) TO RESIN CEMENT
}

\author{
Marwa Emam* and Ahmed Mohamed Arafa**
}

\begin{abstract}
Purpose: This study was aimed to evaluate the effect of repeated heat pressing and thermocycling on micro-shear bond strength of PEEK to resin cement.

Methods: A total of 30 PEEK (Bredent Gmbh \&Co.KG, Germany) specimens $10 \mathrm{~mm}$ x 10 $\mathrm{mm} \times 2 \mathrm{~mm}$ were fabricated and divided to three groups $(\mathrm{n}=10)$. Group I was pressed using new PEEK, Group II; 50\% new PEEK and 50\% reprocessed PEEK, and Group III; 100\% reprocessed PEEK. Bonding surfaces were sandblasted and thin layer of bonding agent (visio.link, Bredent Gmbh \& Co.KG, Germany) was applied and polymerized. Plastic tygon tubes with a $1.5 \mathrm{~mm}$ length and $1 \mathrm{~mm}$ inner diameter were fixed on the sample surface and filled with dual polymerized selfetch self-adhesive resin cement (Totalcem, Itena, Paris, France) and cured for 40 seconds. Half the specimens in each group $(n=5)$ subjected to 5000 thermocycles $\left(5-55^{\circ} \mathrm{C}\right)$ prior to shear bond strength ( $\mu \mathrm{SBS}$ ) test. Two-way Analysis of Variance (ANOVA) was used to analyse the effect of PEEK condition, thermocycling and their interaction on mean micro-shear bond strength $\mu \mathrm{SBS}$. Bonferroni's post-hoc test was used for pair-wise comparisons when ANOVA test is significant. The significance level was set at $\mathrm{P} \leq 0.05$.
\end{abstract}

Results: The PEEK condition (regardless of thermocycling) showed a statistically significant mean $\mu$ SBS value $(\mathrm{P}$-value $=0.001$, Effect size $=0.455)$. Thermocycling (regardless of PEEK condition) also showed a statistically significant mean $\mu$ SBS value (P-value $=0.002$, Effect size $=0.331)$. The interaction between the two variables had no statistically significant effect on mean $\mu$ SBS $($ P-value $=0.442$, Effect size $=0.066)$.

Conclusion: Both repeated heat pressing and thermocycling had a negative effect on microshear bond strength of PEEK to resin cement.

KEYWORDS: PEEK, heat pressing, reprocessing, surface treatment, thermocycling, shear bond strength.

* Lecturer, Department of Fixed Prosthodontics, Faculty of Dentistry, Ain Shams University, Cairo, Egypt.

** Lecturer, Department of Fixed Prosthodontics, Faculty of Dentistry, Beni-Suef University, Beni-Suef, Egypt. 


\section{INTRODUCTION}

Polyetheretherketone (PEEK) is the most significant representative of polyaryletherketone (PAEK) family. It is a partially crystalline, high performance, temperature resistant, thermoplastic material. It consists of an aromatic ring connected by ketones to functional groups ${ }^{(1)}$ with high melting temperature and has numerous applications in industrial processes. PEEK materials are used in medicine, orthopedics and dentistry. A modified PEEK with incorporation of $20 \%$ inorganic fillers are preferred in dental field because of features as stable physical properties, high abrasion resistance, biocompatibility and elastic modulus comparable to bone that allowing it to act as a stress breaker and lessen forces transmitted to restorations and implants ${ }^{(2-4)}$.

Additionally, PEEK is more aesthetic than metal and conventional thermoplastic resins, allowing its use in applications such as implant bodies, temporary abutments, implant superstructures, crowns, telescopic crowns, removable partial dentures and fixed partial dentures ${ }^{(2,5)}$. The low specific weight of PEEK allows it to construct considerably lightweight prosthesis with high comfort and satisfaction of the patient. Its hygienic properties easily helps to maintain oral hygiene ${ }^{(6)}$. Moreover, PEEK is radiolucent material making it compatible with different imaging techniques. This allows the diagnosis, examination, and treatment when necessary without need for substructure removal or impairment ${ }^{(7)}$. BioHPP (High Performance Polymer) is a part of the PEEK family. Its good wear resistance, excellent stability, supreme polishing properties, with resulting low plaque accumulation affinity made it ideal choice for precise prosthetic restorations fabrication ${ }^{(8)}$.

PEEK restorations are constructed either with computer aided design computer aided manufacturing (CAD CAM) or with pressing technology ${ }^{(3)}$. For milling purposes, blanks of PEEK pressed industrially under standardized criteria are supplied while for compression molding, prepressed granular or pellets form is available ${ }^{(9)}$.

The manufacturer instructions warn from pressing the material more than once. They claim that it would degrade during another melting process and important physical and mechanical qualities would be lost ${ }^{(10)}$. After pressing, the sprues should be removed, along with the remaining button material and discarded. New material ingots should be used for new pressings. However, this will result in a significant amount of wasted material and it has been found that remaining materials are being reprocessed in some dental laboratories. The issue was thereby raised whether PEEK material could be safely reprocessed. Sufficient data about microstructure, possible degradation, physical and mechanical properties of dental restorations fabricated from reprocessed material is not available. For industrial purpose, Day et al ${ }^{(1)}$ tested PEEK produced from the blend by injection moulding and found the test pieces to have greater tensile strength and Young's modulus than a new material containing a similar loading of PEEK composites reinforced with carbon fibres. It was also stated that the viscosity molecular weight of the reprocessed PEEK did not drop greatly after an extra injection moulding cycle.

PEEK blanks have an opaque color, greyish or white and are unsuitable for esthetic monolithic dental restorations, especially for the anterior esthetic region. Therefore, veneering is mandatory, but bonding the veneering composite resin materials to PEEK remains bothersome because of its inert chemical performance, low surface energy, poor wetting capabilities and resistance to surface alteration by chemical treatments ${ }^{(12)}$. Some studies evaluated the adhesion of resin cements to treated and untreated PEEK surfaces and the others tested and compared different surface treatments; sandblasting with alumina and silica coating (Rocatec), chemical 
TABLE (1): Materials and equipments used.

\begin{tabular}{cccc}
\hline Material & Product name & Manufacturer & Composition \\
\hline PEEK & $\begin{array}{c}\text { for 2 press BioHPP } \\
\text { (Granulate })\end{array}$ & Bredent GmbH \& Co KG & PEEK, 20\% weight titanium oxide \\
\hline Investment material & Brevest for 2 Press & Bredent GmbH \& Co KG & Phosphate bonded investment \\
\hline Adhesive system & Visio.link & Bredent GmbH \& Co KG & $\begin{array}{c}\text { MMA, pentaerythritol triacrylate, photo } \\
\text { initiators }\end{array}$ \\
\hline Resin cement & Totalcem & Itena & $\begin{array}{c}\text { Self-etching and self-adhesive } \\
\text { Permanent Nanohybrid Composite resin cement }\end{array}$ \\
\hline Aluminium oxide & Cobra & Renfert GmbH & $\begin{array}{c}\text { Aluminium oxide sand (110 } \mu \mathrm{m} \text { mean } \\
\text { particle size })\end{array}$ \\
\hline Sandblaster & $\begin{array}{c}\text { Basic Classic, 70- } \\
250 \mu \mathrm{m}, 220-240 \mathrm{~V}\end{array}$ & Renfert GmbH & 1 x 70-250 $\mu$ m, incl. nozzle 1.2 mm \\
\hline Polymerizing unit & Bre.lux Power Unit 2 & Bredent GmbH \& Co KG & LED Light 370-500 nm \\
\hline
\end{tabular}

treatment with $98 \%$ sulfuric acid, gas inert plasma treatment, silane agents and adhesives. Based on the results, airborne-particle abrasion and an adhesive system could be recommended for reliable bond strength between PEEK substructures and composite resins ${ }^{(5,13-19)}$. The cementation protocol recommended by manufacturer is to induce microroughness using sandblasting with $110 \mu \mathrm{m}$ alumina particles followed by a special adhesive layer application (visio.link, Bredent Gmbh \& Co.KG, Germany) prior to luting system application. Several testing methodologies can be used to assess the bonding properties including shear bond and tensile bond strength tests. As better stress distribution can be fulfilled in smaller specimens, lately more accurate test methods, such as microtensile and microshear tests were introduced ${ }^{(20)}$. To obtain clinically relevant statements, specimens were exposed to artificial aging in a thermocycler. The aim of this study was to assess the effect of repeated heat pressing (reprocessing) of PEEK on the bond strength with resin cement. As well as to describe the failure modes in newly pressed, partially reprocessed and totally reprocessed material using scanning electron microscopy SEM.

\section{MATERIALS AND METHODS}

The details of the materials used in this study are mentioned in Table 1. A total of 30 PEEK specimens (Bredent Gmbh \&Co.KG, Germany) each measuring $10 \mathrm{~mm} \times 10 \mathrm{~mm} \times 2 \mathrm{~mm}$ were constructed. A pink wax sheet of $2 \mathrm{~mm}$ thickness was cut into identical rectangular blocks $10 \mathrm{~mm}$ x $10 \mathrm{~mm}$. The wax patterns were subjected to spruing, investing with phosphate bonded investment (Brevest for 2 press investment material, Bredent, Senden, Germany) and divided to three groups $(\mathrm{n}=10)$. Group I was pressed using new PEEK, Group II ; 50\% new PEEK and 50\% reprocessed PEEK, and Group III ; 100\% reprocessed PEEK. Half the specimens in each group $(n=5)$ submitted through 5000 thermocycles $\left(5-55^{\circ} \mathrm{C}\right)$ before performing shear bond strength ( $\mu$ SBS) test. (Table:2).

TABLE (2): Classification of PEEK specimens in test groups

\begin{tabular}{lc}
\hline \multicolumn{1}{c}{ PEEK composition } & Thermocycling \\
\hline \multirow{2}{*}{ PEEK Control $(\mathbf{n}=\mathbf{1 0})$} & No Thermocycling $(\mathrm{n}=5)$ \\
\cline { 2 - 2 } & Thermocycling $(\mathrm{n}=5)$ \\
\hline PEEK Partially & No Thermocycling $(\mathrm{n}=5)$ \\
\cline { 2 - 2 } reprocessed $(\mathbf{n}=\mathbf{1 0})$ & Thermocycling $(\mathrm{n}=5)$ \\
\hline PEEK Totally & No Thermocycling $(\mathrm{n}=5)$ \\
\cline { 2 - 2 } reprocessed $(\mathbf{n}=\mathbf{1 0})$ & Thermocycling $(\mathrm{n}=5)$ \\
\hline
\end{tabular}


After pressing, the samples were divested then the bonding surfaces of the specimens were polished under running water with 600 and 800 grit silicon carbide paper. To fit the test device, A polyvinyl chloride PVC tube with $25 \mathrm{~mm}$ internal diameter for each specimen was filled with autopolymerising acrylic resin (Cold cure special tray material, Acrostone, Cairo, Egypt) and the PEEK specimens were fixed and lodged in the acrylic resin with the bonding surface exposed and in same level with the edge of the PVC tube. Then, the specimens were carefully washed in an ultrasonic cleaner (CD-4820, CODYSON, Guangdong, China) for 10 minutes with distilled water. finally, the specimens were air dried. All bonding surfaces of PEEK received the same surface treatment; sandblasting with $110 \mu \mathrm{m}$ $\mathrm{Al}_{2} \mathrm{O}_{3}$ at $2.5 \mathrm{bar}$ at $10 \mathrm{~cm}$ distance from the nozzle. Thin , uniform layer of visio.link bonding agent (Bredent Gmbh \&Co.KG, Germany) was applied and polymerized at $220 \mathrm{~mW} / \mathrm{cm} 2$ for 90 seconds (Brelux Power Unit; bredent). Plastic tygon tubes with $1.5 \mathrm{~mm}$ length and $1 \mathrm{~mm}$ inner diameter were fixed on the sample treated surface where the tube axis was perpendicular to sample surface, filled with automix dual polymerized self etch self adhesive resin cement (Totalcem, Itena, Paris, France). Small disposable brushes were used for excess cement removal from the bonding margin using. The cement material was cured using a light-curing unit (3M ESPE Dental Products, St Paul, USA) for 40 seconds.

Half the samples in every group were stored for 24 hours in distilled water at $37^{\circ} \mathrm{C}$. An automated thermocycling machine (100 SD Mechatronic Thermocycler, Germany) was used to expose the other half to 5000 thermocycles (between 5 and $55^{\circ} \mathrm{C}$ ) with a dwell time of 20 second- and 10 -seconds resting time in between by using before the $\mu \mathrm{SBS}$ test. Before testing, samples were checked with light microscope (MA100, Nikon, Japan) at 30x magnification to exclude samples with gaps or air bubbles at PEEK/cement interface. Differences in $\mu$ SBS between PEEK specimens and resin cement were tested with different composition and thermocycling as variables. For shear bond testing, samples were mounted in lower fixed head of a universal testing machine (Instron 3345, Instron Corporation, England) where the bonding interface of PEEK and resin cement perpendicular to the horizontal plane and A stainless- steel wire (diameter: 0.14 inch) attached to the upper movable head of the testing machine applying shear force at the interface with a crosshead speed of $1.0 \mathrm{~mm} / \mathrm{min}$ till specimen failure. Machine software (BlueHill 3 , Instron, England) was used to calculate $\mu \mathrm{SBS}$ (MPa) by dividing the maximum load $(\mathrm{N})$ by the area of the bonding interface $\left(\mathrm{mm}^{2}\right)$.

\section{Failure Analysis}

The samples were carefully inspected using a digital microscope (Dino-Lite Pro, Olympus, Tokyo, Japan). To classify failure occurred, images of the PEEK bonded surfaces and resin cement from each specimen were captured, Image identifiers were removed, and images were examined. The failure mode founded was classified as follows: adhesive PEEK/cement (no resin cement remnants on the PEEK surface), cohesive in cement (the fracture was in the cement) and mixed (remnants of resin cement partially found on the PEEK with PEEK surface exposed).

\section{Scanning Electron Microscopy (SEM)}

One sample from each group was selected, removing the epoxy resin material used for fixation of PEEK to enable topographical analysis of the bonding surface. The bonding surfaces of PEEK were cleaned with isopropyl alcohol, gold-sputtered then evaluated using scanning electron microscopy (SEM) in high vacuum (JSM-6360LA; JEOL, Tokyo, Japan) operating at $15 \mathrm{kV}$. Magnification ranged between 50X and 5000X.

Statistical software (SPSS v.23; IBM, Armonk, NY) was used for performing statistical analysis. Numerical data were investigated for normality by checking the distribution of data and using tests of 
normality (Kolmogorov-Smirnov and Shapiro-Wilk tests). Data showed normal distribution. Data were introduced as mean and standard deviation (SD) values. Two-way Analysis of Variance (ANOVA) was used to assess the effect of PEEK condition, thermocycling and their interaction on mean microshear bond strength. Bonferroni's post-hoc test was used for pair-wise comparisons when ANOVA test is significant. The significance level was set at $\mathrm{P} \leq 0.05$.

\section{RESULTS}

\section{Two-way ANOVA results}

The results revealed that PEEK condition (regardless of thermocycling) showed a statistically significant effect on mean micro-shear bond strength $(\mathrm{P}$-value $=0.001$, Effect size $=0.455)$. Thermocycling (regardless of PEEK condition) also showed a statistically significant effect on mean micro-shear bond strength $(\mathrm{P}$-value $=0.002$, Effect size $=0.331)$. The interaction between the two studied variables had no statistically significant effect on mean micro-shear bond strength (P-value $=0.442$, Effect size $=0.066)$. As the interaction between the variables is non-statistically significant, so the variables are independent from each other. (Table.3)

\section{Effect of PEEK condition regardless of thermocycling}

Regardless of thermocycling; A statistically significant difference was found between mean micro-shear bond strengths of different PEEK conditions $(\mathrm{P}$-value $=0.001$, Effect size $=0.455)$. Pair-wise comparisons revealed that new PEEK showed the statistically significantly highest mean micro-shear bond strength. There was no statistically significant difference between partially and totally reprocessed PEEK; both showed statistically significantly lower mean values. (Table.4) (Fig.1)

TABLE (3) Two-way ANOVA results for the effect of PEEK condition and thermocycling on mean micro-shear bond strength

\begin{tabular}{lcccccc}
\hline Variable & $\begin{array}{c}\text { Type III Sum } \\
\text { of Squares }\end{array}$ & df & Mean Square & F-value & P-value & $\begin{array}{c}\text { Effect size (Partial } \\
\text { eta squared) }\end{array}$ \\
\hline PEEK condition & 97.326 & 2 & 48.663 & 10.029 & $0.001 *$ & 0.455 \\
Thermocycling & 57.561 & 1 & 57.561 & 11.863 & $0.002 *$ & 0.331 \\
PEEK condition $x$ Thermocycling interaction & 8.207 & 2 & 4.104 & 0.846 & 0.442 & 0.066 \\
\hline
\end{tabular}

df: degrees of freedom $=(n-1), *$ : Significant at $P \leq 0.05$

TABLE (4) The mean, standard deviation (SD) values and two-way ANOVA test results for comparison between micro-shear bond strength $(\mathrm{MPa})$ of different PEEK conditions regardless of thermocycling

\begin{tabular}{|c|c|c|c|c|c|c|c|}
\hline \multicolumn{2}{|c|}{$\begin{array}{c}\text { New PEEK } \\
(\mathrm{n}=5)\end{array}$} & \multicolumn{2}{|c|}{$\begin{array}{l}\text { Partially reprocessed } \\
\text { PEEK }(\mathrm{n}=5)\end{array}$} & \multicolumn{2}{|c|}{$\begin{array}{l}\text { Totally reprocessed } \\
\text { PEEK }(\mathrm{n}=5)\end{array}$} & \multirow{2}{*}{ P-value } & \multirow{2}{*}{$\begin{array}{c}\text { Effect size (Partial eta } \\
\text { squared) }\end{array}$} \\
\hline Mean & SD & Mean & SD & Mean & SD & & \\
\hline $15.56^{\mathrm{A}}$ & 2.82 & $12.53^{\text {в }}$ & 2.32 & $11.26^{\mathrm{B}}$ & 2.63 & $0.001 *$ & 0.455 \\
\hline
\end{tabular}

*: Significant at $P \leq 0.05$, Different superscripts are statistically significantly different 


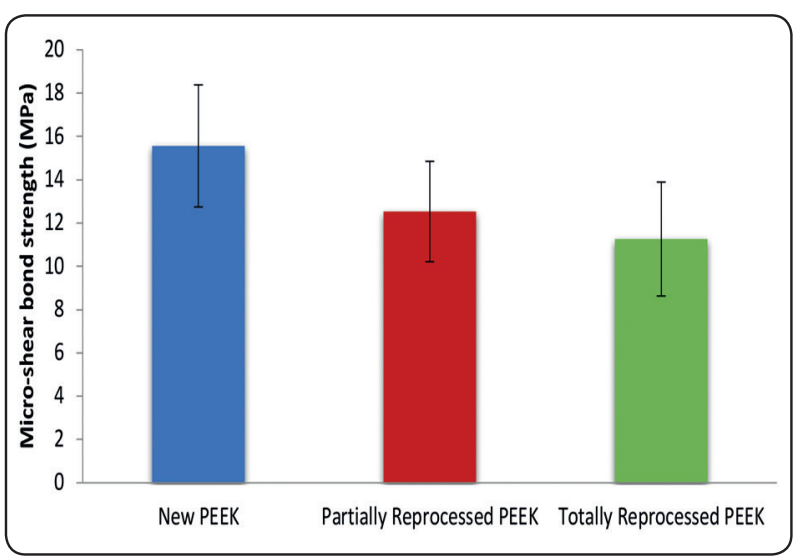

Fig. (1) Bar chart representing mean and standard deviation values for micro-shear bond strength of different PEEK conditions regardless of thermocycling

Effect of thermocycling regardless of PEEK condition

Regardless of PEEK condition; non-thermocycled specimens revealed statistically significant higher mean micro-shear bond strength than thermocycled specimens $(\mathrm{P}$-value $=0.002$, Effect size $=$ 0.331). (Table.5) (Fig.2)

TABLE (5) The mean, standard deviation (SD) values and two-way ANOVA test results for comparison between micro-shear bond strength (MPa) of non-thermocycled and thermocycled specimens regardless of PEEK condition

\begin{tabular}{cccccc}
\hline \multicolumn{2}{c}{$\begin{array}{c}\text { Non- } \\
\text { thermocycled }\end{array}$} & Thermocycled & P-value & $\begin{array}{c}\text { Effect size } \\
\text { (Partial eta } \\
\text { squared) }\end{array}$ \\
\cline { 1 - 4 } Mean & SD & Mean & SD & & $0.002 *$ \\
\hline 14.5 & 2.3 & 11.73 & 3.25 & 0.331 \\
\hline
\end{tabular}

*: Significant at $P \leq 0.05$

Effect of different interactions on micro-shear bond strength

\section{Comparison between PEEK conditions}

As regards non-thermocycled specimens; statistically significant difference was found between

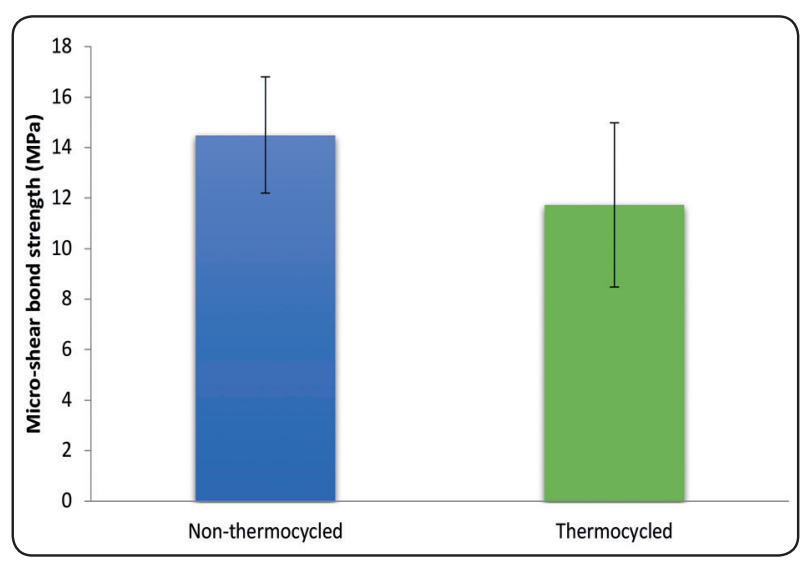

Fig. (2) Bar chart representing mean and standard deviation values for micro-shear bond strength of non-thermocycled and thermocycled specimens regardless of PEEK condition

mean micro-shear bond strength of different PEEK conditions $(\mathrm{P}$-value $=0.100$, Effect size $=0.175)$.

While with thermocycled specimens; statistically significant difference was found between mean micro-shear bond strengths of different PEEK conditions $(\mathrm{P}$-value $=0.002$, Effect size $=0.41$ ) Pair-wise comparisons between conditions revealed that new PEEK showed the statistically significantly highest mean micro-shear bond strength. There was no statistically significa nt difference between partially and totally reprocessed PEEK; both showed statistically significantly lower mean values. (Table 6) (Fig.3)

\section{Comparison between non-thermocycled and thermocycled specimens:}

As regards new PEEK; there was no statistically significant difference between mean micro-shear bond strength of non-thermocycled and thermocycled specimens $(\mathrm{P}$-value $=0.321$, Effect size $=0.041)$.

While with partially as well as totally reprocessed PEEK; non-thermocycled specimens showed statistically significantly higher mean micro- 
TABLE (6). The mean, standard deviation (SD) values and two-way ANOVA test results for comparison between micro-shear bond strength (MPa) with different variables interactions

\begin{tabular}{|c|c|c|c|c|c|c|c|c|}
\hline \multirow{2}{*}{ Thermocycling } & \multicolumn{2}{|c|}{$\begin{array}{c}\text { New PEEK } \\
(\mathrm{n}=5)\end{array}$} & \multicolumn{2}{|c|}{$\begin{array}{c}\text { Partially reprocessed } \\
\text { PEEK }(\mathrm{n}=5)\end{array}$} & \multicolumn{2}{|c|}{$\begin{array}{l}\text { Totally reprocessed } \\
\text { PEEK }(n=5)\end{array}$} & \multirow{2}{*}{ P-value } & \multirow{2}{*}{$\begin{array}{c}\text { Effect size } \\
\text { (Partial eta } \\
\text { squared) }\end{array}$} \\
\hline & Mean & SD & Mean & SD & Mean & SD & & \\
\hline Non-thermocycled & 16.26 & 1.71 & 14.01 & 2.45 & 13.24 & 1.85 & 0.100 & 0.175 \\
\hline Thermocycled & $14.85^{\mathrm{A}}$ & 3.71 & $11.06^{\mathrm{B}}$ & 0.83 & $9.29^{\text {в }}$ & 1.52 & $0.002 *$ & 0.41 \\
\hline P-value & \multicolumn{2}{|c|}{0.321} & \multicolumn{2}{|c|}{$0.045^{*}$} & \multicolumn{2}{|c|}{$0.009^{*}$} & & \\
\hline $\begin{array}{l}\text { Effect size (Partial } \\
\text { eta squared) }\end{array}$ & \multicolumn{2}{|c|}{0.041} & \multicolumn{2}{|c|}{0.157} & \multicolumn{2}{|c|}{0.251} & & \\
\hline
\end{tabular}

*: Significant at $P \leq 0.05$, Different superscripts in the same row indicate statistically significant difference between conditions

shear bond strength than thermocycled specimens $(\mathrm{P}$-value $=0.045$, Effect size $=0.157)$ and $(\mathrm{P}$-value $=0.009$, Effect size $=0.251)$, respectively. $($ Table.6) (Fig.3)

The failure analysis showed that the most common mode of failure was adhesive failure followed by cohesive failure and the least common was mixed type. (Fig.4) SEM evaluations of surface topography at $50 \mathrm{X}$ were used to study failure modes of different groups. (Fig.5-7)

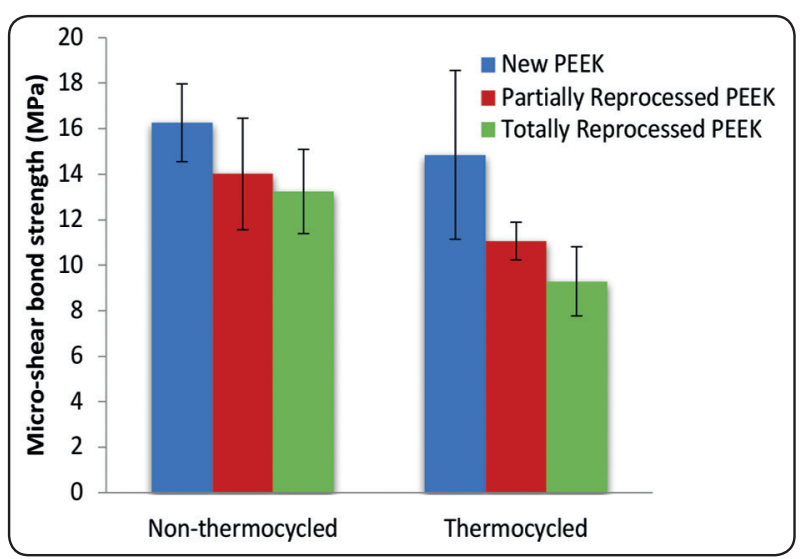

Fig. (3). Bar chart representing mean and standard deviation values for micro-shear bond strength with different interactions of variables

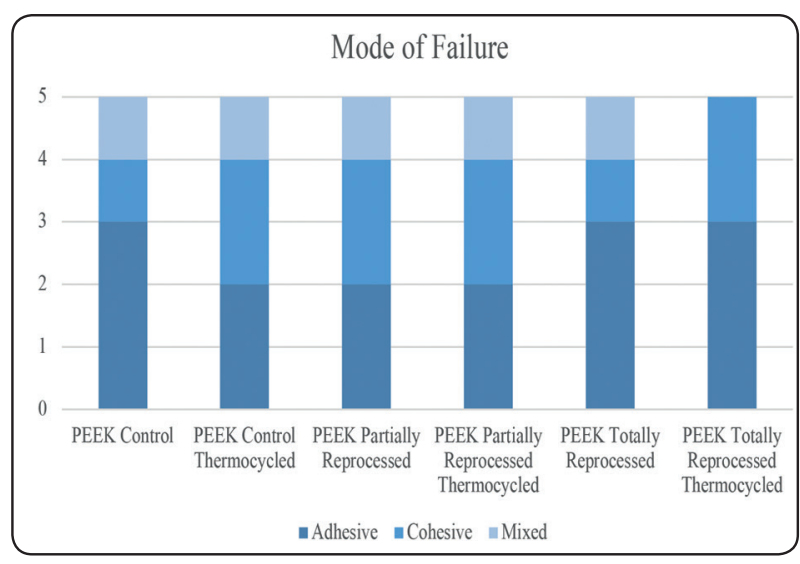

Fig. (4) Bar chart representing mode of failure analysis of all groups.

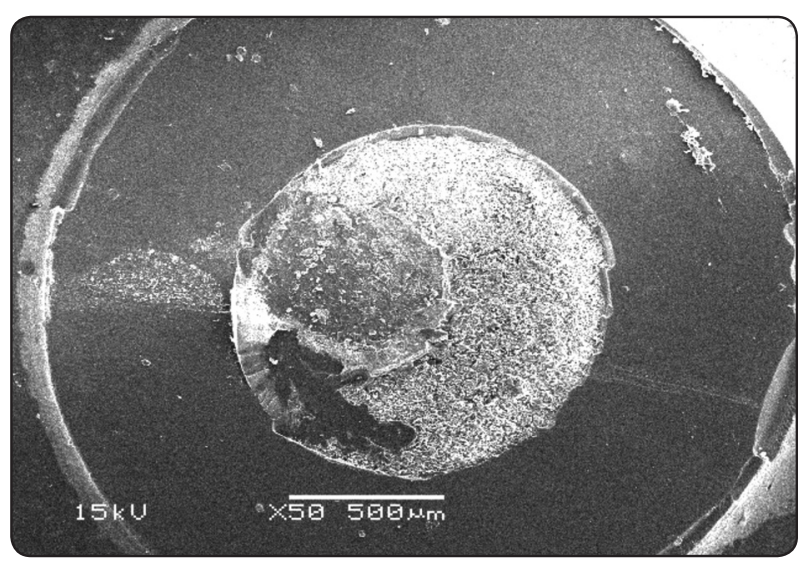

Fig. (5) Topographical analysis of the bonding surface of PEEK partially reprocessed Thermocycled sample showing cohesive mode of failure of cement layer (50X). 


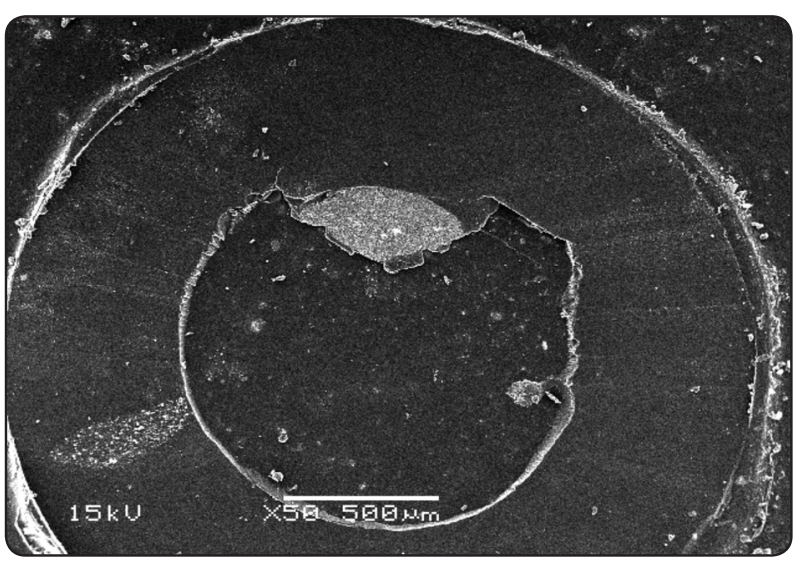

Fig. (6) Topographical analysis of the bonding surface of PEEK totally reprocessed sample showing adhesive mode of failure (50X).

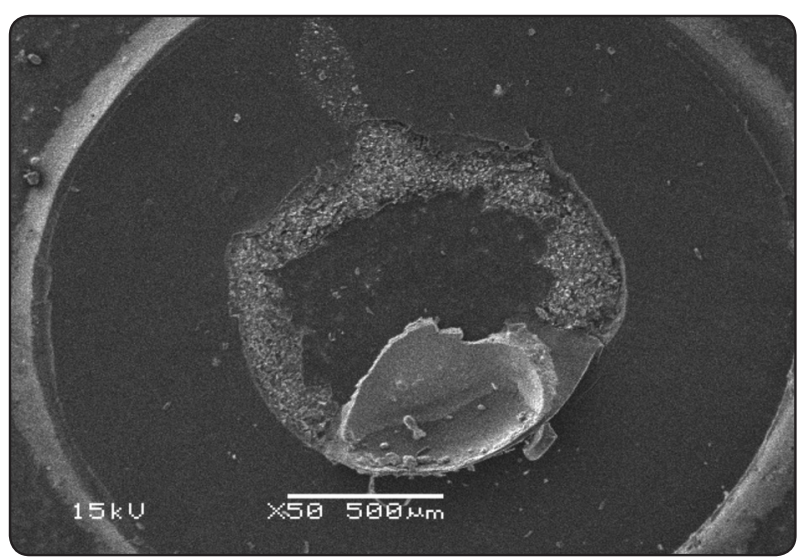

Fig. (7) Topographical analysis of the bonding surface of PEEK totally reprocessed thermocycled sample showing mixed mode of failure $(50 \mathrm{X})$

\section{DISCUSSION}

Based on the mechanical and physical properties, PEEK seems to be an appropriate material for dental prosthesis. However, adequate bonding between PEEK and veneering resins is fundamental in ensuring long survival and success rates ${ }^{(21)}$. It is also obscure whether adherence to PEEK can withstand the hydrolytic effects due to water sorption, which is responsible for reduction of resin-bonding capability to oxide ceramics ${ }^{(19)}$. Surface topography is a fundamental factor that enables mechanical bonding due to the adhesive penetration inside the pits resulting in resin tags formation. Sandblasting creates surface roughness, cleans organic contaminant from the surface leaving active surface layer promoting micromechanical interlocking with resin-based dental materials ${ }^{(4)}$. for enhancing the bonding of resin to inert PEEK surface, dental adhesives are used where the adhesive system's content and solvents are fundamental factors to be considered. According to previous studies, MMA monomers containing adhesive systems showed higher values of bond strength between resin and PEEK (15,19,22-24). It has been recommended to coat PEEK surface with low viscosity adhesive systems before covering it with veneering resins ${ }^{(25,26)}$. For this purpose, Visio.link bond was selected as an adhesive system in this study (4). This study evaluated the effect of repeated heat processing and Thermocycling on bond strength of PEEK to resin cement. The results manifested that incorporation of previously processed PEEK either partially or totally and thermocycling decreased $\mu$ SBS between resin cement and PEEK significantly. To the knowledge of the authors, this is the first study to evaluate the effect of reprocessing of PEEK used for dental restorations on bonding with resin cement. For that reason, the results obtained from this study could not be compared to results obtained from other available studies. The $\mu$ SBS values of both partially and totally reprocessed PEEK were significantly lower than the new PEEK. The crosslinking between functional group of etched/air abraded PEEK and monomer functional groups of adhesive systems was believed to be the cause of the enhancement of bond strength. Oxidative degradation of PEEK was believed to include two reactions, chain scission and cross-linking. Chain scission results in molecular chains with less length and higher mobility, that allows reorganisation of the crystalline structure, on the other hand cross-linking prompt molecular branching and meshing ${ }^{(27)}$. Previous studies ${ }^{(28,29)}$ showed that exposing PEEK to longer times and higher temperatures processing procedures in air induce a lower level of re-crystallised material and concluded that the oxidative reactions turn out to precede cross-linking reactions. 
Consequently, this may lead to diminished number of bonds available for bonding with the adhesive. $\mu$ SBS tests are suitable to evaluate the adhesive efficiency of resin-based materials. Any variations in the properties and features of the evaluated material surface may influence the $\mu$ SBS values, which are attributed to mechanical and chemical adhesion ${ }^{(4)}$. The $\mu$ SBS test was selected for the measurement of bond strength in this study as it is easy to perform and not technique sensitive allowing decreasing the number of pretest failures ${ }^{(30)}$.

As the oral temperatures fluctuate, the longterm bonding stability of PEEK is not guaranteed. In this study, the specimens were thermocycled for 5,000 in a thermocycling machine, approximately corresponds to 6 months of intraoral service in vivo ${ }^{(31)}$. The results showed that thermocycling decreased $\mu$ SBS between resin cement and PEEK. Thermocycling includes repeated exposure to two temperatures $\left(55\right.$ and $5{ }^{\circ} \mathrm{C}$ ) with $20 \mathrm{sec}$ dwell time to guarantee the specimens are not exposed to extreme thermal stresses. The decreased $\mu$ SBS might be accounted for thermal loading leading to mechanical stress bonding interface and leading to volumetric changes. Therefore, cracks can be initiated and propagated along the bonding interface, caused by the different dimensional changes of the materials resulting in decreasing the bond strength values ${ }^{(19)}$. In a study by Stawarczyk et al. ${ }^{(19)}$ it was confirmed that an adhesive application before bonding to a self-adhesive resin cement enabled establishing of bonding after thermocycling even without etching or air abrasion surface treatment. In another study $^{(15)}$ Thermocycling after pretreatment with Visio.link and Signum PEEK adhesives showed no influence on tensile bond strength. On the other hand, thermocycling was proved by other studies to have a detrimental effect on the resin-material bonding due to relaxation of stresses within the composites. These stresses were resulted from polymerization shrinkage process ${ }^{(32,33)}$. A previous study tested the effect of aging conditions on the bond strength of a resin composite to a composite and found that
5000 thermal cycling was the most influential in the degradation of the composite tested between other tested aging methods ${ }^{(34)}$. A previous study ${ }^{(35)}$ evaluated the shear bond strength of adhesive system bonded to different pretreated PEEK surfaces using different thermocycling methods. The bond strength after thermocycling was significantly dropped for all groups. They attributed the degradation to the $55^{\circ} \mathrm{C}$ hot water that might have further stimulated the hydrolysis of incompletely polymerized or unpolymerized resin cements. The failure type's analysis in this study showed no differences in the mode of fracture depending on PEEK reprocessing or thermocycling.

\section{CONCLUSION}

1. PEEK reprocessing had a negative effect on micro-shear bond strength with resin cement as new PEEK showed the highest bond strength.

2. Thermocycling had a negative effect on microshear bond strength of resin cement with both partially and totally reprocessed PEEK.

\section{Clinical recommendation}

1. Only new PEEK should be used for pressing dental restorations as it showed the highest micro-shear bond strength with resin cement even under thermocycling conditions.

2. Using reprocessed PEEK has detrimental effect on its bond strength to resin cement especially after thermocycling.

\section{REFERENCES}

1. Stawarczyk, B, Taufall, S, Roos, M, Schmidlin, P, \& Lümkemann, N. Bonding of composite resins to PEEK: the influence of adhesive systems and air-abrasion parameters. Clinical Oral Investigations 2017; 22(2), 763-771.

2. Kurahashi K, Matsuda T, Ishida Y, Ichikawa T. Effect of Surface Treatments on Shear Bond Strength of Polyetheretherketone to Autopolymerizing Resin. Dent J (Basel). 2019; 1;7(3):82. 
3. Atsü SS, Aksan ME, Bulut AC. Fracture Resistance of Titanium, Zirconia, and Ceramic-Reinforced Polyetheretherketone Implant Abutments Supporting CAD/CAM Monolithic Lithium Disilicate Ceramic Crowns After Aging. Int J Oral Maxillofac Implants. 2019; 34(3):622-630.

4. Caglar I, Ates SM, Yesil Duymus Z. An In Vitro Evaluation of the Effect of Various Adhesives and Surface Treatments on Bond Strength of Resin Cement to Polyetheretherketone. J Prosthodont. 2019; 28(1):e342-e349.

5. Rocha, R., Anami, L., Campos, T., Melo, R., Souza, R., \& Bottino, M. Bonding of the Polymer Polyetheretherketone (PEEK) to Human Dentin: Effect of Surface Treatments. Brazilian Dental Journal, 2016; 27(6), 693-699.

6. Bathala, L., Majeti, V., Rachuri, N., Singh, N., \& Gedela, S. The Role of Polyether Ether Ketone (Peek) in Dentistry - A Review. Journal Of Medicine And Life, 2019; 12(1), 5-9.

7. Zhou, L., Qian, Y., Zhu, Y., Liu, H., Gan, K., \& Guo, J. The effect of different surface treatments on the bond strength of PEEK composite materials. Dental Materials, 2014; 30(8), e209-e215.

8. Georgiev J, Vlahova A, Kissov H, Aleksandrov S, Kazakova R. Possible application of BioHPP in prosthetic dentistry: a literature review. J of IMAB. 2018 ;24(1):1896-1898.

9. Stawarczyk B, Eichberger M, Uhrenbacher J, Wimmer T, Edelhoff D, Schmidlin PR. Three-unit reinforced polyetheretherketone composite FDPs: influence of fabrication method on load-bearing capacity and failure types. Dent Mater J. 2015;34(1):7-12.

10. Bredent. BioHPP in for 2 press system, processing instructions

11. Day, R.J., Wood, A.K., Pang, S.F. Recycling of APC-2 offcuts. Composites Manufacturing 1994; 5 (3), 187-193.

12. Stawarczyk B, Thrun H, Eichberger M, Roos M, Edelhoff D, Schweiger J, Schmidlin PR. Effect of different surface pretreatments and adhesives on the load-bearing capacity of veneered 3-unit PEEK FDPs. J Prosthet Dent. 2015;114(5):666-73

13. Uhrenbacher J, Schmidlin PR, Keul C, Eichberger M, Roos M, Gernet W, Stawarczyk B. The effect of surface modification on the retention strength of polyetheretherketone crowns adhesively bonded to dentin abutments. J Prosthet Dent. 2014;112(6):1489-97.

14. Gama LT, Duque TM, Özcan M, Philippi AG, Mezzomo LAM, Gonçalves TMSV. Adhesion to high-performance polymers applied in dentistry: A systematic review. Dent Mater. 2020; 36(4):e93-e108.

15. Stawarczyk B, Keul C, Beuer F, Roos M, Schmidlin PR. Tensile bond strength of veneering resins to PEEK: impact of different adhesives. Dent Mater J. 2013;32(3):441-8

16. Sproesser, O., Schmidlin, P., Uhrenbacher, J., Eichberger, M., Roos, M., \& Stawarczyk, B. Work of adhesion between resin composite cements and PEEK as a function of etching duration with sulfuric acid and its correlation with bond strength values. International Journal Of Adhesion And Adhesives. 2014; 54, 184-190.

17. Sproesser O, Schmidlin PR, Uhrenbacher J, Roos M, Gernet W, Stawarczyk B. Effect of sulfuric acid etching of polyetheretherketone on the shear bond strength to resin cements. J Adhes Dent. 2014; 16:465-472.

18. Bähr N, Keul C, Edelhoff D, Eichberger M, Roos M, Gernet W, Stawarczyk B. Effect of different adhesives combined with two resin composite cements on shear bond strength to polymeric CAD/CAM materials. Dent Mater J. 2013; 32:492-501.

19. Stawarczyk B, Bähr N, Beuer F, Wimmer T, Eichberger M, Gernet W, Jahn D, Schmidlin PR. Influence of plasma pretreatment on shear bond strength of self-adhesive resin cements to polyetheretherketone. Clin Oral Investig. 2014; 18(1):163-70.

20. Andrade AM, Moura SK, Reis A, Loguercio AD, Garcia EJ, Grande RH. Evaluating resin-enamel bonds by microshear and microtensile bond strength tests: effects of composite resin. J Appl Oral Sci. 2010; 18(6):591-8.

21. Stawarczyk, B., Jordan, P., Schmidlin, P., Roos, M., Eichberger, M., Gernet, W., \& Keul, C. PEEK surface treatment effects on tensile bond strength to veneering resins. J Prosthet Dent.. 2014; 112(5), 1278-1288.

22. Rosentritt M, Preis V, Behr M, Sereno N, Kolbeck C. Shear bond strength between veneering composite and PEEK after different surface modifications. Clin Oral Investig. 2015;19(3):739-44.

23. Keul C, Liebermann A, Schmidlin PR, Roos M, Sener B, Stawarczyk B. Influence of PEEK surface modification on surface properties and bond strength to veneering resin composites. J Adhes Dent. 2014; 16(4):383-92.

24. Silthampitag $\mathrm{P}$, Chaijareenont $\mathrm{P}$, Tattakorn K, Banjongprasert C, Takahashi H, Arksornnukit M. Effect of surface pretreatments on resin composite bonding to PEEK. Dent Mater J. 2016;35(4):668-74. 
25. Kern M, Lehmann F. Influence of surface conditioning on bonding to polyetheretherketon (PEEK). Dent Mater. 2012; 28(12):1280-3.

26. Chaijareenont P, Prakhamsai S, Silthampitag P, Takahashi H, Arksornnukit M. Effects of different sulfuric acid etching concentrations on PEEK surface bonding to resin composite. Dent Mater J. 2018 8;37(3):385-392.

27. McLauchlin, A., Ghita, O., \& Savage, L. Studies on the reprocessability of poly(ether ether ketone) (PEEK). J. Mater. Process. Technol., 2014; 214 (1), 75-80.

28. Jones, D., Leach, D., \& Moore, D. (1985). Mechanical properties of poly(ether-ether-ketone) for engineering applications. Polymer, 26(9), 1385-1393.

29. Day, M., Sally, D., \& Wiles, D. Thermal degradation of poly(aryl-ether-ether-ketone): Experimental evaluation of crosslinking reactions. Journal Of Applied Polymer Science. 1990; 40(910), 1615-1625.

30. Bumrungruan, C., \& Sakoolnamarka, R. Microshear bond strength to dentin of self-adhesive flowable composite compared with total-etch and all-in-one adhesives. Journal Of Dental Sciences. 2016; 11(4), 449-456.
31. Stewardson DA, Shortall AC, Marquis PM. The effect of clinically relevant thermocycling on the flexural properties of endodontic post materials. J Dent. 2010; 38(5):437-42.

32. Xie C, Han Y, Zhao XY, Wang ZY, He HM. Microtensile bond strength of one- and two-step self-etching adhesives on sclerotic dentin: the effects of thermocycling. Oper Dent. 2010; 35(5):547-55.

33. Nikaido T, Kunzelmann KH, Chen H, Ogata M, Harada N, Yamaguchi S, Cox CF, Hickel R, Tagami J. Evaluation of thermal cycling and mechanical loading on bond strength of a self-etching primer system to dentin. Dent Mater. 2002; 18(3):269-75.

34. Ozcan M, Barbosa SH, Melo RM, Galhano GA, Bottino MA. Effect of surface conditioning methods on the microtensile bond strength of resin composite to composite after aging conditions. Dent Mater. 2007; 23(10):1276-82.

35. Zhou L, Qian Y, Gan K, Liu H, Liu X, Niu D. Effect of different surface treatments and thermocycling on shear bond strength to polyetheretherketone. High Performance Polymers. 2017;29(1):87-93. 